\title{
Verschärftes rechnerisches Verfahren der harmonischen Analyse.
}

\author{
Von
}

\section{Walter Dällenbach.}

\section{Einleitung.}

Ist eine periodische Funktion der Zeit $f(t)$ im ganzen Periodenintervall $o$ bis $\mathrm{T}$ durch die Fourierreihe

$$
\left.\begin{array}{c}
f(t)=\frac{a_{0}}{2}+a_{1} \cos \omega t+b_{1} \sin \omega t+a_{2} \cos (2 \omega t)+b_{2} \sin (2 \omega t+\ldots \ldots \\
+a_{n} \cos (n \omega t)+b_{n} \sin (n \omega t)+\ldots \text { in inf. }
\end{array}\right\}
$$

darstellbar, so berechnen sich deren Koeffizienten $z u$

$$
\begin{aligned}
& a_{n}=\frac{2}{T} \int_{0}^{T} f(t) \cos (n \omega t) d t \\
& b_{n}=\frac{2}{T} \int_{0}^{T} f(t) \sin (n \omega t) d t
\end{aligned}
$$

$\omega=2 \pi \nu=\frac{2 \pi}{\mathrm{T}}$ ist die Kreisfrequenz der Grundwelle. Die Funktion $\mathrm{f}(\mathrm{t})$ sei empirisch durch eine Kurve gegeben. Dann besteht eine angenäherte Berechnung der Koeffizienten $a_{n}$ und $b_{n}$ darin, daß man das Intervall o bis $T$ in $m$ gleiche Teilintervalle der Öffnung $\frac{\mathrm{I}}{\mathrm{m}}$ einteilt und die Integrale für $\mathrm{a}_{\mathrm{n}}$ und $b_{\mathrm{n}}$ durch die Summen approximiert

$$
\left.\begin{array}{l}
\mathrm{a}_{\mathrm{n}}^{*}=\frac{2}{\mathrm{~m}} \sum_{\mathrm{a}=\mathrm{x}}^{\mathrm{m}} \mathrm{f}_{\mathrm{a}} \cos _{\mathrm{a}} \\
\mathrm{b}_{\mathrm{n}}=\frac{2}{\mathrm{~m}} \sum_{\mathrm{a}=\mathrm{r}}^{\mathrm{m}} \mathrm{f}_{\mathrm{a}} \sin _{\mathrm{a}} .
\end{array}\right\}
$$

$f_{a}, \cos _{a}, \sin _{a}$ bedeuten richtig bezogen die Werte der Funktionen $f(t), \cos (n \omega t)$, $\sin (n \omega t)$ im $a^{\text {ten }}$ Teilpunkt. Dieses angenäherte Verfahren der harmonischen Analyse wollen wir das Rungesche nennen, denn Runge beschreibt eine zur numerischen Rechnung bequeme Handhabung davon ${ }^{1}$ ). Es hat den Nachteil, daß auch bei ziemlich glatt verlaufenden Kurven die Anzahl m der Teilpunkte groß gewählt werden muß, wenn die Werte der 7,8,9 und höherer Harmonischen noch einigermaßen stimmen sollen. Ferner liefert es keine anschauliche Vorstellung vom Fehler, den man begeht. Denn bei Annäherung der Integrale durch Summen, ist es ja nicht die zu analysierende Originalkurve $f(t)$, sondern es sind die nicht direkt gegebenen Funktionen $f(t) \cdot \cos (n \omega t)$ und $f(t) \cdot \sin (n \omega t)$, die durch $m$-stufige Treppenkurven ersetzt werden.

Anläßlich einiger Analysen, die ich in einer elektrotechnischen Frage durchzuführen die Gelegenheit hatte, habe ich dem Mathematiker Herrn Prof. Hermann

1) Vgl. Runge, Theorie und Praxis der Reihen. Sammlung Schubert XXXII (I904) $\S 17$. Wiedergegeben in Hütte, Des Ingenieurs Taschenbuch (I92I), Band I, Seite I3o. 
Weyl die Vermutung geäußert, es sollte doch möglich sein, das kungesche Verfahren in ähnlicher Weise zu verschärfen, wie etwa die Simpsonsche Regel eine Verschärfung der Annäherung eines einfachen Integrals durch eine Summe darstellt. Herr Weyl hat dann die im nächsten Abschnitt wiedergegebene Annäherung gerechnet. Sie liefert die strenge Analyse des Polygonzuges, der entsteht, wenn die Bogen zwischen aufeinanderfolgenden Teilpunkten der Originalkurve durch die Sehnen ersetzt werden. Im darauffolgenden Abschnitt endlich habe ich die Annäherung weiter getrieben. Durch vier aufeinanderfolgende Teilpunkte etwa $(a-2, a-I, a, a+I)$ wird die kubische Parabel gelegt. Ersetzt man die Originalkurve nur im mittleren Intervall $(a-I, a)$ durch den kubischen Parabelbogen und das für alle Intervalle $\mathrm{a}=\mathrm{I}$ bis $\mathrm{m}$, so erhält man auch bei verhältnismäßig geringer Anzahl von Teilpunkten eine Ersatzkurve, die sich mit einer für die Bedürfnisse des Technikers ausreichenden Genauigkeit an eine einigermaßen glatt verlaufende Originalkurve anschmiegt. Diese aus $m$ kubischen Parabelbögen zusammengestückelte Ersatzkurve wird streng analysiert. Es rechtfertigt sich, hier darüber zu berichten, da die Ergebnisse einfach und praktisch sind. Sowohl im Fall des Polygonzuges, wie im Fall der kubischen Parabelbögen als Ersatzkurve, sind die nach Runge berechneten Werte $a_{n}^{*}$ und $b_{n}^{*} z u \cdot$ multiplizieren mit einem in den zwei Fällen verschiedenen Faktor $\mathrm{K}$, der allein abhängt von der Nummer $\mathrm{n}$ der Harmonischen und der Anzahl $\mathrm{m}$ der Teilpunkte. Die Korrektur ist für die höheren Harmonischen beträchtlich und beträgt z. B. bei Annäherung durch kubische Parabelbogen und bei $m=24$ Teilpunkten für die $3^{\text {te }}$ Harmonische $1 \%$, für die $5^{\text {te }} \cdot 4 \%$, für die $7^{\text {te }}$ I $2 \%$, für die $9^{\text {te }} 27 \%$ und für die $\mathrm{II}^{\text {te }} 46 \%$.

\section{Annäherung durch Polygonzug.}

Um die Rechnung einfacher zu gestalten, benützen wir die Schreibweise in komplexen Zahlen $(\mathrm{j}=\sqrt{-\mathrm{I}})$.

$$
f(t)=\sum_{-\infty}^{+\infty} c_{n} e^{j n \omega t}
$$

ist eine mit (I) völlig identische Darstellung der Fourierreihe, falls die komplexen Koeffizienten vermöge

$$
c_{n}=\frac{I}{2}\left(a_{n}-j b_{n}\right) \text { und } c_{-n}=\frac{1}{2}\left(a_{n}+j b_{n}\right)
$$

mit den reellen $a_{n}$ und $b_{n}$ zusammenhängen ${ }^{1}$ ).

An Stelle von (2) tritt dann

$$
c_{n}=\frac{I}{T} \int_{0}^{T} f(t) e^{-j n \omega t} d t .
$$

Sein angenäherter Wert nach dem Runge schen Verfahren beträgt entsprechend (3)

$$
c_{n}^{*}=\frac{1}{m} \sum_{a=1}^{m} f_{a} e_{a},
$$

wo $f_{a}$ und $e_{a}$ die Werte von $f(t)$ und $e^{-j n \omega t}$ im $a^{\text {ten }}$ Teilpunkte bedeuten.

Mit der aus Bild I ersichtlichen Bezeichnung ist im a ${ }^{\text {ten }}$ "Teilintervall

$$
\mathrm{f}(\mathrm{t})=\mathrm{f}(\boldsymbol{\vartheta})=\mathrm{f}_{\mathrm{a}-\mathrm{x}}+\left(\mathrm{f}_{\mathrm{a}}-\mathrm{f}_{\mathrm{a}-\mathrm{r}}\right) \frac{\boldsymbol{\vartheta}}{\Theta}+\eta
$$

1) Vergleiche hierüber z. B. Burkhardt, Einführung in die Theorie der analytischen Funktionen einer komplexen Veränderlichen (1903) $\$ 49$. 
$\eta$ ist die Abweichung der Originalkurve vom Polygonzug. Bis auf den Rest

$$
\begin{gathered}
R=\frac{I}{T} \int_{t=0}^{T} \eta e^{-j n(t)} d t \quad \text { ist } \\
c_{n}=\sum_{a=I}^{m} c_{n a}, \text { wo } \\
c_{n a}=\frac{I}{T} \int_{\mathfrak{l}=0}^{\Theta}\left[f_{a-x}+\left(f_{a}-f_{a-r}\right) \frac{\vartheta}{\Theta}\right] e^{-j n \omega[(a-r) \Theta+\vartheta]} d \mathfrak{g}
\end{gathered}
$$

den Beitrag des $a^{\text {ten }}$ Teilintervalls zum Werte von $c_{n}$ bedeutet.

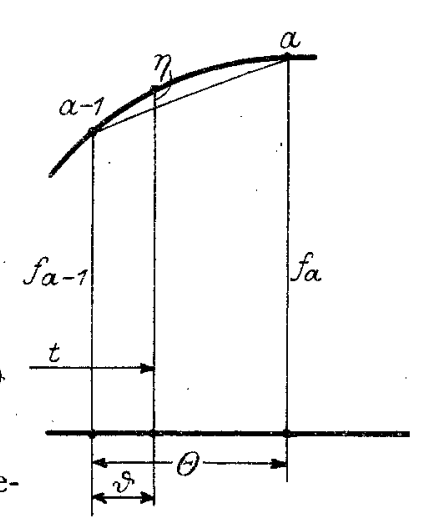

Bild I.

$$
c_{n a}=\frac{-1}{j 2 \pi n}\left[f_{a-r}\left(e_{a}-e_{a-r}\right)+\left(f_{a}-f_{a-r}\right) \int_{0}^{\Theta} \frac{\vartheta}{\Theta} d\left\{e^{-j n \omega[(a-1) \Theta+\vartheta]}\right\}\right] .
$$

Das verbleibende Integral partiell integriert

$$
c_{n a}=\frac{-I}{j 2 \pi n}\left\{f_{a} e_{a}-f_{a-r} e_{a-x}+\frac{m}{j 2 \pi n}\left(f_{a}-f_{a}-r\right)\left(e_{a}-e_{a-r}\right)\right\} .
$$

Also ist bis auf den angegebenen Rest $R$

$$
c_{n}=\frac{m}{(2 \pi n)^{2}} \sum_{a=I}^{m}\left(f_{a}-f_{a-1}\right)\left(e_{a}-e_{a-I}\right) .
$$

Ordnet man diese Summe nach den Funktionswerten $f_{a}$, so erhält $f_{a}$ den Faktor:

$$
\begin{aligned}
& 2 \mathrm{e}_{\mathrm{a}}-\mathrm{e}_{\mathrm{a}-\mathrm{I}}-\mathrm{e}_{\mathrm{a}+\mathrm{I}}=\mathrm{e}_{\mathrm{a}}\left(2-\mathrm{e}_{-\mathrm{I}}-\mathrm{e}_{+\mathrm{I}}\right) \\
& =\mathrm{e}_{\mathrm{a}}\left(2-\mathrm{e}^{\mathrm{j} 2 \pi \frac{\mathrm{n}}{\mathrm{m}}}-\mathrm{e}^{-\mathrm{j} 2 \pi \frac{\mathrm{n}}{\mathrm{m}}}\right) \\
& =2 \mathrm{e}_{\mathrm{a}}\left[\mathrm{I}-\dot{\cos }\left(2 \pi \frac{\mathrm{n}}{\mathrm{m}}\right)\right] \\
& =4 e_{a} \sin ^{2}\left(\pi \frac{n}{m}\right) \text {. }
\end{aligned}
$$

Folglich

$$
c_{\mathrm{n}}=\left[\frac{\sin \frac{\pi \mathrm{n}}{\mathrm{m}}}{\frac{\pi \mathrm{n}}{\mathrm{m}}}\right]^{2} \cdot \frac{1}{\mathrm{~m}} \sum_{\mathrm{a}=\mathrm{I}}^{\mathrm{m}} \mathrm{f}_{\mathrm{a}} \mathrm{e}_{\mathrm{a}}
$$

oder streng

$$
c_{n}=K c_{n}^{*}+R \text {. }
$$

Da

$$
\left|\mathrm{e}^{-j \mathrm{n} \omega \mathrm{t}}\right|=\mathbf{I} \quad \text { ist } \quad \mathrm{R} \leqq \frac{\mathrm{I}}{\mathbf{T}} \int_{0}^{\mathrm{T}}|\boldsymbol{\eta}| \mathrm{dt}=\overline{|\eta|}
$$

Es ist also endgültig

oder für die reellen Koeffizienten

$$
c_{n} \leqq K c_{n}^{*}+\overline{|\eta|}
$$

$$
a_{n} \leqq K a_{n}^{*}+\overline{|\eta|}
$$




$$
\mathrm{b}_{\mathrm{n}} \leqq K \mathrm{~b}_{\mathrm{n}}^{*}+\eta_{i}
$$

Hierin bedeuten $\eta$ den Mittelwert des absoluten Betrages der Abweichung des Polygonzuges von der Originalkurve, gebildet über das Periodenintervall o bis $T$,

$$
\mathrm{K}=\left[\begin{array}{c}
\sin \frac{\pi \mathrm{n}}{\mathrm{m}} \\
-\pi \mathrm{n} \\
\mathrm{m}
\end{array}\right]^{2}
$$

der Korrektionsfaktor, gebaut wie das Quadrat eines Wicklungsfaktors, der allein abhängt von der Anzahl. $m$ der Teilintervalle und der Nummer $\mathrm{n}$ der Harmonischen. $c_{n}^{*}$ bzw. $a_{n}^{*}$ und $b_{n}^{*}$ sind die nach dem Rungeschen Verfahren, Formeln (3) und (6), ermittelten Koeffizienten der Fourierreihe.

\section{Annäherung durch kubische Parabelbogen.}

Mit den aus Bild 2 ersichtlichen Bezeichnungen ist im Intervall vom $(a-2)^{\text {ten }}$ bis zum $(a+I)^{\text {ten }}$ Teilpunkt

$$
\mathrm{f}(\mathrm{t})=\mathrm{f}(\boldsymbol{\vartheta})=\mathrm{A} \boldsymbol{\vartheta}^{3}+\mathrm{B} \vartheta^{2}+\mathrm{C} \vartheta+\mathrm{D}+\eta \text {. }
$$

$\eta$ ist die Abweichung der Originalkurve von der kubischen Parabel, die durch die vier aufeinanderfolgenden Teilpunkte vom $(a-2)^{\text {ten }}$ bis zum $\left(a+{ }^{\circ} \mathrm{I}\right)^{\text {ten }}$ hindurchgeht. Die Konstanten A, B, C, D haben also den Gleichungen zu genügen:

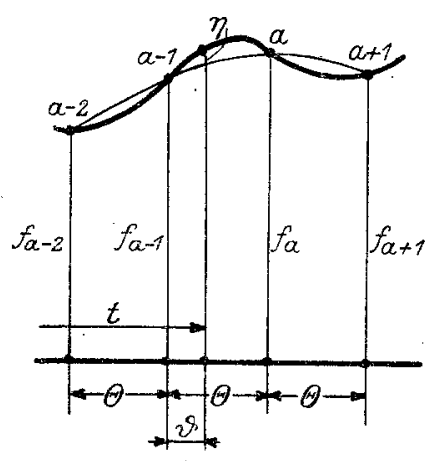

Bild 2.

$$
\begin{aligned}
-A \Theta^{3}+B \Theta^{2}-C \Theta+D & =f_{a-a} \\
D & =f_{a-r} \\
A \Theta^{3}+B \Theta^{2}+C \Theta+D & =f_{2}
\end{aligned}
$$$$
8 A \Theta^{3}+4 B \Theta^{2}+2 C \Theta+D=f_{a}+I
$$

und bestimmen sich daraus $z \mathrm{u}$

$$
\begin{aligned}
& A_{1}=6 \Theta^{3} A=f_{a+1}-3 f_{a}+3 f_{2-x}-f_{a-2} \\
& B_{1}=2 \Theta^{2} B=\quad f_{a}-2 f_{a-x}+f_{a-2} \\
& C_{1}=6 \Theta C=-f_{a+x}+6 f_{a}-3 f_{a-x}-2 f_{a-2} \\
& D=\quad f_{a-r .}
\end{aligned}
$$

Bis auf den von der Abweichung $\eta$ herrührenden Rest ist daher bei obiger Zerlegung von $f(t)$ der Beitrag des $a^{\text {ten }}$ Teilintervalls zum Werte von $c_{n}$.

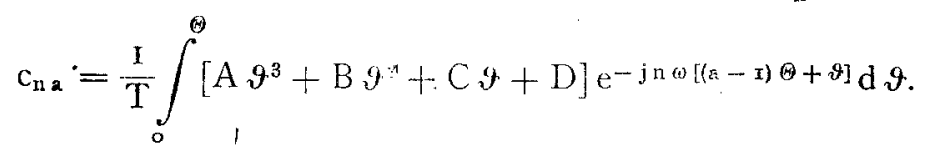

Ersetzt man $A, B, C$ durch $A_{1}, B_{1}, C_{1}$, substituiert $u=\frac{9}{\Theta}$ als neue Variable und nennt $-\mathrm{j} 2 \pi \frac{\mathrm{n}}{\mathrm{m}}=\frac{\mathrm{I}}{\lambda}$, so resultiert

$$
c_{n a}=\frac{e_{a}-x}{m} \int_{0}^{I}\left[\frac{I}{6} A_{1} u^{3}+\frac{I}{2} B_{1} u^{2}+\frac{I}{6} c_{1} u+D\right] e^{\frac{u}{\lambda}} d u .
$$

Nun betragen die Integrale

$$
\begin{aligned}
& \int_{0}^{x} e^{\frac{u}{\lambda}} d u=\lambda\left(e^{\frac{x}{\lambda}}-I\right) \\
& \int_{0}^{x} u e^{\frac{u}{\lambda}} d u=\lambda\left[(I-\lambda) e^{\frac{x}{\lambda}}+\lambda\right]
\end{aligned}
$$




$$
\begin{aligned}
& \int_{0}^{I} u^{2} e^{\frac{u}{\lambda}} d u=\lambda\left[\left(I-2 \lambda+2 \lambda^{2}\right) \mathrm{e}^{\frac{I}{\lambda}}-2 \lambda^{2}\right] \\
& \int_{0}^{I} u^{3} e^{\frac{u}{\lambda}} d u=\lambda\left[\left(I-3 \lambda+6 \lambda^{2}-6 \lambda^{3}\right) \mathrm{e}^{\frac{1}{\lambda}}+6 \lambda^{3}\right] .
\end{aligned}
$$

Führt man diese Werte in $c_{n a}$ ein, berücksichtigt, daß $\mathrm{e}^{\frac{x}{\lambda}}=\mathrm{e}_{1} ; \mathrm{e}^{-\frac{\mathrm{x}}{\hat{\lambda}}}=\mathrm{e}_{-\mathrm{x}}$; $\mathrm{e}_{1} \mathrm{e}_{\mathrm{a}-\mathrm{r}}=\mathrm{e}_{\mathrm{a}}$ und ordnet nach Potenzen von $\lambda$, so wird

$$
\begin{gathered}
c_{n a}=\lambda \frac{e_{a}}{m}\left\{\left[\left(\frac{I}{6} A_{1}+\frac{I}{2} B_{1}+\frac{I}{6} C_{1}+D\right)-e_{-x} D\right]+\left[-\frac{I}{2} A_{1}-B_{1}-\frac{I}{6} C_{1}+\frac{I}{6} e_{-I} c_{1}\right] \lambda+\right. \\
\left.+\left[A_{1}+B_{1}-e_{-x} B_{1}\right] \lambda^{2}+\left[-A_{1}+e_{-x} A_{1}\right] \lambda^{3}\right\} .
\end{gathered}
$$

Setzt man die Ausdrücke für $A_{1}, B_{1}, C_{1}, D$ ein, bildet $c_{n}=\sum_{a=I}^{m} c_{n a}+$ Rest und ordnet die Summe nach $f_{a}$, so ergibt sich

wo

$$
c_{n} \leqq K \frac{I}{m} \sum_{a=I}^{m} f_{a} e_{a}+\overline{|\eta|}
$$

oder als Ergebnis

$$
\begin{aligned}
\mathrm{K} & =\lambda^{2}\left(\lambda^{2}-\frac{\mathrm{I}}{6}\right)\left[\mathrm{e}_{2}+\mathrm{e}_{-2}-4\left(\mathrm{e}_{1}+\mathrm{e}_{-1}\right)+6\right] \\
& =\lambda^{2}\left(\lambda^{2}-\frac{1}{6}\right)\left[\mathrm{e}^{\mathrm{j} \pi \frac{\mathrm{n}}{\mathrm{m}}-\mathrm{e}^{\mathrm{j} x} \mathrm{~m}}\right]^{*}
\end{aligned}
$$

$$
\begin{aligned}
& c_{n} \leqq K c_{n}^{*}+r \mid \\
& a_{n} \leqq K a_{n}^{*}+\overline{|r|} \\
& b_{n} \leqq K b_{n}^{*}+\mid \overline{\eta_{1}},
\end{aligned}
$$

wo $\bar{\eta}, c_{n}^{*}, a_{n}^{*}, b_{n}^{*}$ die gleiche Bedeutung haben wie im vorigen Abschnitt und

$$
\mathrm{K}=\left[1+\frac{2}{3}\left(\frac{\pi \mathrm{n}}{\mathrm{m}}\right)^{2}\right]\left[\begin{array}{c}
\sin \frac{\pi \mathrm{n}}{\mathrm{m}} \\
\frac{\pi \mathrm{n}}{\mathrm{m}}
\end{array}\right]^{4}
$$

der nur von $m$ und $\mathrm{n}$ abhängige Korrektionsfaktor ist.

Für $m=24$ Teilpunkte sind die Werte von $K$ bis zur $\mathrm{I}_{2}{ }^{\text {ten }}$ Harmonischen ${ }^{1}$ )

\begin{tabular}{c|c|c|c}
\hline $\mathrm{n}$ & $\mathrm{K}$ & $\mathrm{n}$ & $\mathrm{K}$ \\
\hline $\mathbf{I}$ & 0,99993 & 7 & 0,87654 \\
$\mathbf{2}$ & 0,99888 & 8 & 0,80970 \\
3 & 0,99453 & 9 & 0,72816 \\
4 & 0,98353 & I0 & 0,63519 \\
5 & 0,96217 & II & 0,53546 \\
6 & $0,9272 \mathrm{I}$ & I2 & 0,43445
\end{tabular}

1) Den Studenten der Eidgen. Techn. Hochschule, die mir bei der Kontrolle dieser kleinen Tabelle behilflich waren, danke ich. 
Sie lassen sich benützen zur Verschärfung, der Analyse nach dem Rechenchema, wie es angegeben ist in der Hütte, Band I, Seite I32, Abschnitt II.

\section{Schlufbemerkungen.}

I. Hat die zu analysierende Kurve im groben einen ziemlich glatten Verlauf, dem aber eine intensive Oberwelle überlagert ist, wie das etwa bei Feldkurven mit ausgeprägten Nutenoberschwingungen vorkommt, so ist es zweckmäßig, diese Oberwelle zu schätzen, von der Originalkurve graphisch zu subtrahieren und erst die verbleibende glatter verlaufende Differenzkurve rechnerisch zu analysieren.

II. Es mag mathematisches Interesse bieten, die in dieser Arbeit angegebene Methode fortzusetzen und durch $\mathrm{k}$ aufeinanderfolgende Teilpunkte die Parabel $(\mathrm{k}-\mathrm{I})^{\text {ten }}$ Grades zu legen, - den Grenzfall $\mathrm{k}=\mathrm{m}$ eingeschlossen - und zu sehen, welches bei geeigneter Wahl der Teilintegrationsintervalle der allgemeine Ausdruck ist, den der Korrektionsfaktor $\mathrm{K}$ annimmt. Eine numerisch bessere Näherung ist davon kaum zu erwarten.

III. Beim Durchsehen der Literatur finde ich, daß Runge ${ }^{1}$ ) einen ähnlichen Versuch gemacht hat. Er legt als Ersatzkurve durch zwei aufeinanderfolgende Teilpunkte eine Parabel $\mathrm{k}^{\text {ten }}$ Grades und verlangt, daß aneinanderstoßende Parabelbogen in den Teilpunkten auch noch in den Ableitungen bis zu einer Ordnung übereinstimmen sollen, die genügt, die Parabelbogen eindeutig festzulegen. Es führt das zu Summen für $c_{n}$, in welche die Funktionswerte $f^{a}$ wie bei der Simpsonschen Regel mit verschiedenem Gewicht eingehen, so daß $c_{n}$ sich nicht mehr aus $c_{n}^{*}$ berechnen läßt. Die Handhabung ist nicht mehr einfach.

IV. Außer rechnerischen Verfahren der harmonischen Analyse ist noch das graphische Verfahren von Fischer-Hinnen (ETZ. I90I, Seite 396) ziemlich bekannt. Theoretisch, d. h. wenn man die mit vielfachem Abzirkeln von Strecken verbundenen Felder vernachläßigt, ergibt es genaue und nicht bloß angenäherte Werte der Oberwellen. Umständlich ist, daß das Intervall zur Bestimmung jeder Oberwelle immer anders eingeteilt werden muß. Man kann hierzu eine kleine Teilmaschine benützen. 'Das Verfahren verlangt ferner, daß die höchsten Oberwellen, die gerade noch von merkbarem Einfluß sind auf die empirisch gegebene Kurve, zuerst bestimmt werden. Aus diesen folgen dann rekursiv diejenigen niedrigerer Ordnung. Die Grundwelle erhält man zuletzt. Man ist also gezwungen, die Analyse immer - auch dann, wenn nur die Oberwellen niedrigerer Ordnung interessieren bis zu der Genauigkeit durchzuführen, mit der die zu analysierende Kurve gegeben ist.

Ich habe gefunden, daß ich mit dem beschriebenen, verschärften $R$ ungeschen Verfahren, das sich kubischer Parabelbögen zur Annäherung bedient, in Aufgaben der Elektrotechnik rascher und genauer zum Ziel komme, als mit irgend einem andern mir bekannten rechnerischen oder graphischen Verfahren.

Baden (Schweiz), im Juli 192 I.

1) Runge, a. a. O., $\$ 20$, Seite I92 bis 196. 